\title{
The levels of HDAC1 and thioredoxin1 are related to the death of mesothelioma cells by suberoylanilide hydroxamic acid
}

\author{
BO RA YOU and WOO HYUN PARK \\ Department of Physiology, Medical School, Institute for Medical Sciences, \\ Chonbuk National University, Jeonju 561-180, Republic of Korea
}

Received December 31, 2015; Accepted February 11, 2016

DOI: $10.3892 /$ ijo.2016.3402

\begin{abstract}
Mesothelioma is an aggressive tumor which is mainly derived from the pleura of lung. In the present study, we evaluated the anticancer effect of suberoylanilide hydroxamic acid (SAHA), a histone deacetylase (HDAC) inhibitor on human mesothelioma cells in relation to the levels of $\mathrm{HDACl}$, reactive oxygen species (ROS) and thioredoxin (Trx). While $1 \mu \mathrm{M}$ SAHA inhibited cell growth in Phi and ROB cells at $24 \mathrm{~h}$, it did not affect the growth in ADA and Mill cells. Notably, the level of HDAC1 was relatively overexpressed among Phi, REN and ROB cells. SAHA induced necrosis and apoptosis, which was accompanied by the cleavages of PARP and caspase-3 in Phi cells. This agent also increased the loss of mitochondrial membrane potential (MMP, $\Delta \Psi \mathrm{m}$ ) in Phi cells. All the tested caspase inhibitors attenuated apoptosis in SAHA-treated Phi cells whereas HDAC1 siRNA enhanced the apoptotic cell death. SAHA increased intracellular ROS levels including $\mathrm{O}_{2}{ }^{-}$ in Phi cells. N-acetyl cysteine (NAC) and vitamin C (Vit.C) significantly reduced the growth inhibition and death of Phi cells caused by SAHA. This drug decreased the mRNA and protein levels of Trx1 in Phi and ROB cells. Furthermore, Trx1 siRNA increased cell death and $\mathrm{O}_{2}{ }^{--}$level in SAHA-treated Phi cells. In conclusion, SAHA selectively inhibited the growth of Phi and ROB mesothelioma cells, which showed the higher basal level of HDAC1. SAHA-induced Phi cell death was related to oxidative stress and Trx1 levels.
\end{abstract}

Correspondence to: Professor Woo Hyun Park, Department of Physiology, Medical School, Chonbuk National University, Jeonju 561-180, Republic of Korea

E-mail: parkwh71@chonbuk.ac.kr

Abbreviations: SAHA, suberoylanilide hydroxamic acid; HAT, histone acetyltransferase; HDAC, histone deacetylase; ROS, reactive oxygen species; FITC, fluorescein isothiocyanate; MMP $(\Delta \Psi \mathrm{m})$, mitochondrial membrane potential; NAC, $\mathrm{N}$-acetylcysteine; Vit.C, vitamin C; LDH, lactate dehydrogenase; $\mathrm{H}_{2}$ DCFDA, 2',7'-dichlorodihydrofluorescein diacetate; DHE, dihydroethidium; Trx, thioredoxin; siRNA, small interfering RNA

Key words: mesothelioma, histone deacetylase, suberoylanilide hydroxamic acid, thioredoxin, reactive oxygen species

\section{Introduction}

Mesothelioma is a rare malignant tumor and arises in the pleura of lung, peritoneum and pericardium (1). It is important to study malignant mesothelioma because most patients with mesothelioma have worked in careers such as mining. Many environmental factors such as erionite and asbestos are related to carcinogenesis of mesothelioma (2). Especially, asbestos can be easily inhaled or ingested and it is collected in mesothelial tissue, and thereby asbestos fibers cause cellular damage that result in tumor growth (3). Furthermore, asbestos influences epigenetic status of mesothelioma (4). Despite treatment with chemotherapy and radiation therapy, it has a poor prognosis.

Acetylation and deacetylation on histones are controlled by two enzymes, histone acetyltransferase (HAT) and histone deacetylase (HDAC), respectively. The aberrant histone acetylation by the imbalance between HAT and HDAC can lead to carcinogenesis in many cancer cells including colon and mesothelioma $(5,6)$. Especially, HDAC1 overexpression promotes invasion and cell proliferation in cancer cells of the prostate and ovary $(7,8)$. Emerging evidence has demonstrated that HDAC inhibitors are a potent therapeutic agent for treatment of mesothelioma $(9,10)$.

Reactive oxygen species (ROS) have important roles in gene expression, cell signaling and cell differentiation (11). However, excessive ROS production may result in significant damage to cells through oxidizing DNA, proteins and lipids. For this reason, there are various antioxidants in the cells. Thioredoxin1 ( $\operatorname{Trx} 1)$, as a small antioxidant protein increased in mesothelioma (12). It is also demonstrated that asbestos modulates ROS level and the redox status of Trx and it finally affects carcinogenesis of mesothelioma $(13,14)$. Moreover, many studies report that $\operatorname{Trx} 1$ is a target molecule for drugresistance and therapeutics of cancer $(15,16)$.

Suberoylanilide hydroxamic acid (SAHA), a first HDAC inhibitor for cutaneous $\mathrm{T}$ cell lymphoma treatment has an anticancer effect in diverse cancer cells $(17,18)$. SAHA induced apoptosis via FLICE-like inhibitory protein (FLIP)/caspase-8 activation in mesothelioma cells (19). However, little is known about the molecular mechanism of mesothelioma cell death caused by SAHA in view of the levels of HDAC1, ROS and Trx1. Therefore, in the present study we investigated the effects of SAHA on cell death in various mesothelioma cells with regard to HDAC1, ROS and Trx1 levels. 


\section{Materials and methods}

Cell culture. Human mesothelial cells (HM69 and HM72) and human mesothelioma cells (ADA, CON, Hmeso, Mill, Phi, REN and ROB) were obtained from the University of Hawaii Cancer Center (Honolulu, HI, USA). These cells were cultured in Ham's F-12 media containing 10\% fetal bovine serum (FBS; Gibco-BRL, Grand Island, NY, USA) and $1 \%$ penicillinstreptomycin (Gibco-BRL).

Reagents. SAHA purchased from Cayman Chemical Co., (Ann Arbor, MI, USA) was dissolved in dimethyl sulfoxide (DMSO; Sigma-Aldrich, St. Louis, MO, USA). The pan-caspase inhibitor (Z-VAD-FMK), caspase-3 inhibitor (Z-DEVD-FMK), caspase-8 inhibitor (Z-IETD-FMK) and caspase-9 inhibitor (Z-LEHD-FMK) were obtained from R\&D Systems (Minneapolis, MN, USA) and were dissolved in DMSO. NAC and Vit.C obtained from Sigma-Aldrich Chemical were dissolved in $20 \mathrm{mM}$ HEPES (pH 7.0) buffer and water, respectively. Based on previous studies $(20,21)$, cells were pretreated with $15 \mu \mathrm{M}$ caspase inhibitors, $2 \mathrm{mM}$ NAC or $0.4 \mathrm{mM}$ Vit.C for $1 \mathrm{~h}$ before SAHA treatment.

Growth inhibition assay. The effect of SAHA on growth inhibition in human mesothelioma cells was determined by measuring the absorbance of 3-(4,5-dimethylthiazol-2-yl)2,5-diphenyltetrazolium bromide (MTT; Sigma-Aldrich) dye absorbance as previously described (22). Briefly, $5 \times 10^{3}$ cells were incubated with the indicated concentrations of SAHA with or without each caspase inhibitor, HDAC siRNA, NAC, Vit.C or Trx1 siRNA for $24 \mathrm{~h}$.

Western blot analysis. The protein expression levels were evaluated by western blot analysis. Briefly, $1 \times 10^{6}$ cells were incubated with $5 \mu \mathrm{M}$ SAHA for $24 \mathrm{~h}$. Total protein $(30 \mu \mathrm{g})$ was resolved by 4-20\% SDS-PAGE gels, and then transferred to Immobilon-P PVDF membranes (Merck Millipore, Darmstadt, Germany) by electroblotting. Then membranes were probed with anti-HDAC1, anti-acetylated H4, anti-PARP, anti-cleaved PARP, anti-cleaved caspase-3 (Cell Signaling Technology, Danvers, MA, USA), anti-Trx1, anti-GAPDH and anti- $\beta$-actin (Santa Cruz Biotechnology, Santa Cruz, CA, USA). Membranes were incubated with fluorescenceconjugated secondary antibodies.

Measurement of HDAC activity. The HDAC activity was measured by using a HDAC assay kit according to the manufacturer's instructions (Merck Millipore). Briefly, 1x10 cells were incubated with $5 \mu \mathrm{M}$ SAHA for $24 \mathrm{~h}$. Total protein $(30 \mu \mathrm{g})$ was used to measure the HDAC activity.

Annexin V-FITC/PI staining for apoptosis detection. Apoptosis was detected by staining cells with Annexin V-fluorescein isothiocyanate (FITC; Invitrogen-Life Technologies, Eugene, OR, USA; Ex/Em=488 nm/519 nm) and propidium iodide (PI; Sigma-Aldrich; Ex/Em=488 $\mathrm{nm} / 617 \mathrm{~nm}$ ) as previously described (23). Briefly, $1 \times 10^{6}$ cells were incubated with the indicated concentrations of SAHA with or without each caspase inhibitor, NAC, Vit.C, HDAC1 and Trx 1 siRNAs for $24 \mathrm{~h}$. Annexin V/PI staining was analyzed with the Accuri C6 flow cytometer (BD Biosciences, Franklin Lakes, NJ, USA).

Measurement of lactate dehydrogenase (LDH) activity. Necrosis in cells was evaluated by an LDH kit according to the manufacturer's instructions (Sigma-Aldrich). Briefly, $1 \times 10^{6}$ cells were incubated with $5 \mu \mathrm{M}$ SAHA with or without each caspase inhibitor, NAC or Vit.C for $24 \mathrm{~h}$. LDH release was expressed as the percent of extracellular LDH activity compared with the control cells.

Measurement of MMP $\left(\Delta \Psi_{m}\right)$. MMP $(\Delta \Psi \mathrm{m})$ levels were measured using JC-1 dyes (Enzo Life Sciences, Plymouth Metting, PA, USA; Ex/Em=515 nm/529 nm). Briefly, 5x10 cells were incubated with $5 \mu \mathrm{M}$ SAHA for $24 \mathrm{~h}$. Cells were washed twice with PBS and incubated with $10 \mu \mathrm{g} / \mathrm{ml} \mathrm{JC}-1$ at $37^{\circ} \mathrm{C}$ for $30 \mathrm{~min}$. Then cells were washed three times with PBS and analyzed with the Accuri C6 flow cytometer (BD Biosciences). Green fluorescence indicates a monomeric state at the low $\Delta \Psi \mathrm{m}$ and red fluorescence presents an aggregate state at the high $\Delta \Psi_{\mathrm{m}}$.

Sub-G1 analysis. Sub-G1 analysis was determined by PI (Sigma-Aldrich) staining as previously described (23). Briefly, $1 \times 10^{6}$ cells were incubated with $5 \mu \mathrm{M}$ SAHA with or without each caspase inhibitor, HDAC1 and Trx1 siRNAs for $24 \mathrm{~h}$. Sub-G1 DNA content cells were measured and analyzed with Accuri C6 flow cytometer (BD Biosciences).

Transfection of cells with HDACl and Trx1 siRNAs. Gene silencing of HDAC1 and Trx1 was performed by using small interference RNA (siRNA) delivery system. A non-specific control siRNA duplex [5'-CCUACGCCACCAAUUUCGU (dTdT)-3'], HDAC1 siRNA duplex [5'-GAGUCAAAACAGA GGAUGA(dTdT)-3'] and Trx1 siRNA duplex [5'-GCAUGCC AACAUUCCAGUU(dTdT)-3'] were purchased from the Bioneer Corp. (Daejeon, South Korea). In brief, $2.5 \times 10^{5}$ cells were incubated in RPMI-1640 supplemented with 10\% FBS. The next day, cells ( $30-40 \%$ confluence) in each well were transfected with the control, HDAC1 siRNA or Trx1 siRNA [100 pmol in Opti-MEM (Gibco-BRL)] using Lipofectamine 2000 according to the manufacturer's instructions (InvitrogenLife Technologies). One day later, cells were treated with or without $5 \mu \mathrm{M}$ SAHA for additional $24 \mathrm{~h}$. The transfected cells were collected and used for western blot analysis, cell growth, sub-G1, Annexin V-FITC and $\mathrm{O}_{2}{ }^{\circ-}$ level measurements.

Detection of intracellular ROS levels. Intracellular ROS were detected by a fluorescent probe dye, 2',7'-dichlorodihydrofluorescein diacetate $\left(\mathrm{H}_{2} \mathrm{DCFDA}, \mathrm{Ex} / \mathrm{Em}=495 \mathrm{~nm} / 529 \mathrm{~nm}\right.$; Invitrogen-Molecular Probes) as previously described (23). Dihydroethidium (DHE, Ex/Em=518 nm/605 nm; InvitrogenMolecular Probes) is a fluorogenic probe that is highly selective for $\mathrm{O}_{2}{ }^{--}$among ROS. Briefly, $1 \times 10^{6}$ cells were incubated with $5 \mu \mathrm{M}$ SAHA with or without Trx 1 siRNA for $24 \mathrm{~h}$. DCF and DHE fluorescence were detected by using the Accuri C6 flow cytometer (BD Biosciences).

Real-time PCR analysis. Total RNA was extracted by using E.Z.N.A. Total RNA kit (Omega Bio-Tek Inc., Norcross, GA, 


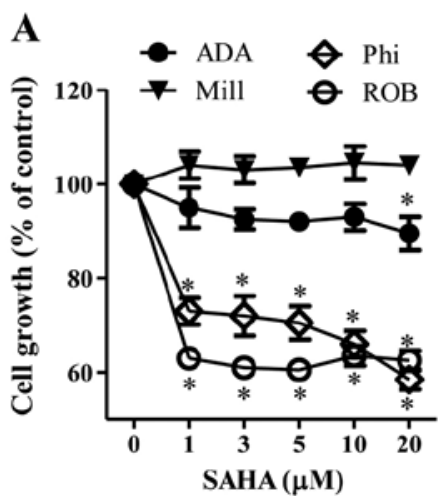

C

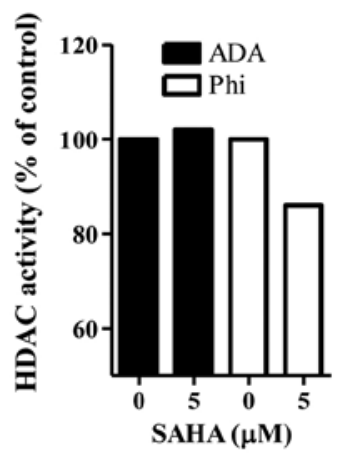

$\mathbf{B}$

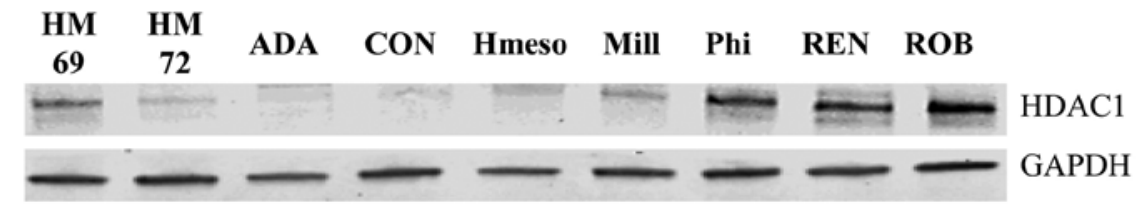

D

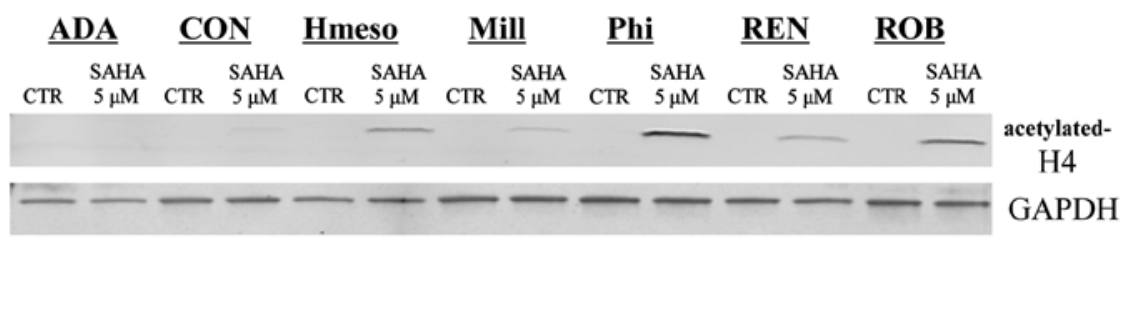

Figure 1. Effects of SAHA on cell growth and HDAC activities in human mesothelioma cells. (A) Graph shows cell growth. (B) The expression level of HDAC1 was examined by western blot analysis. (C) Graph shows HDAC activities in ADA and Phi cells. (D) The expression of acetylated H4 in mesothelioma cells. ${ }^{*} \mathrm{P}<0.05$ compared with SAHA-untreated control group.

USA) according to the manufacturer's instruction. cDNA was obtained from $0.8 \mu \mathrm{g}$ of total RNA by using High-Capacity cDNA reverse transcription kit (Life Technologies). Real-time PCR was performed using a SYBR-Green SuperMix (Quanta Bioscience, Gaithersburg, MD, USA) in real-time PCR cycler, LightCyclerR 480 instrument (Roche Diagnostics, Mannheim, Germany), setting the cycles as follows: $10 \mathrm{~min} / 95^{\circ} \mathrm{C} \mathrm{PCR}$ initial activation step; 40 cycles of denaturation for $15 \mathrm{sec} / 95^{\circ} \mathrm{C}$ and annealing/extenstion step for $25 \mathrm{sec} / 58^{\circ} \mathrm{C}$. Trx 1 (P115235) and hypoxanthine-guanine phosphoribosyltransferase (HPRT, P160523) were obtained from the Bioneer Corp. The changes in mRNA level were determined by the formula $2^{-\Delta \Delta C T}$. The relative amount of mRNA in the sample was normalized to HPRT mRNA.

Statistical analysis. The results represent the mean of at least three independent experiments (mean \pm SD). The data were analyzed using an Instat software (GraphPad Prism5; GraphPad Software, Inc., San Diego, CA, USA). The Student's t-test or one-way analysis of variance (ANOVA) with post hoc analysis using Tukey's multiple comparison test was used for parametric data. Statistical significance was defined as $\mathrm{P}<0.05$.

\section{Results}

Effects of SAHA on cell growth and HDAC activities in human mesothelioma cells. SAHA inhibited the growth of Phi and ROB mesothelioma cells at $24 \mathrm{~h}$ (Fig. 1A). Treatment with $5 \mu \mathrm{M}$ SAHA reduced the growth of Phi and ROB cells $\sim 30-40 \%$ compared to control cells (Fig. 1A). However, $5 \mu \mathrm{M}$ SAHA did not significantly affect the growth of ADA and Mill cells at $24 \mathrm{~h}$ (Fig. 1A). The basal levels of HDAC1 were increased in Phi, REN and ROB cells, whose cells were sensitive to SAHA (Fig. 1B). ADA and Mill cells resistant to SAHA showed lower levels of HDAC1 (Fig. 1B). The HDAC1 levels were different between the two normal mesothelial cells (Fig. 1B).

After the exposure of mesothelioma cells to SAHA for $24 \mathrm{~h}$, SAHA strongly decreased the activity of HDAC in Phi cells but this agent did not change the activity of HDAC in ADA cells (Fig. 1C). Furthermore, the levels of acetylated-H4 were increased in SAHA-treated Hmeso, Phi, REN and ROB cells (Fig. 1D). However, SAHA did not alter the levels of the acetylated-H4 in ADA, CON and Mill cells (Fig. D).

Effects of SAHA on cell death and mitochondrial membrane potential (MMP; $\Delta \Psi m)$ in ADA and Phi cells. Treatment with $5 \mu \mathrm{M}$ SAHA increased the numbers of Annexin V-FITC cells in Phi cells (Fig. 2A). In addition, SAHA induced the cleavages of PARP and caspase-3 in Phi cells (Fig. 2B). Moreover, this agent significantly increased LDH release in Phi cells (Fig. 2C). These results implied that SAHA-induced Phi cell death occurred via apoptosis as well as necrosis. However, SAHA did not influence the percent of Annexin V-FITC cells, apoptosis-related protein levels and LDH release in ADA cells (Fig. 2A-C). The loss of MMP $(\Delta \Psi \mathrm{m})$ can lead to cell death. As shown in Fig. 2D, red fluorescence of JC-1 indicating the high $\Delta \Psi \mathrm{m}$ was decreased in $5 \mu \mathrm{M}$ SAHA-treated Phi cells whereas green fluorescence of JC-1 indicating the low $\Delta \Psi \mathrm{m}$ was increased in these cells (Fig. 2D). SAHA affected neither red fluorescence nor green fluorescence of JC-1 in ADA cells 


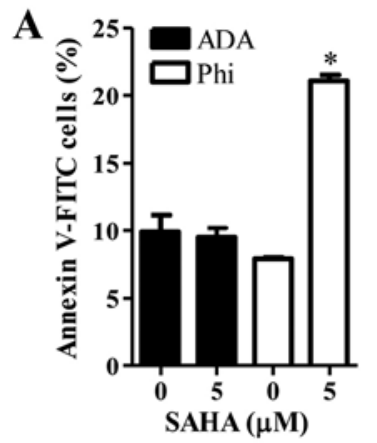

B

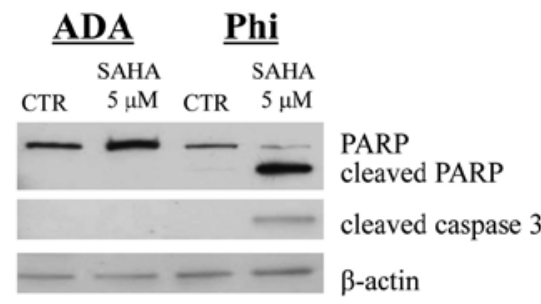

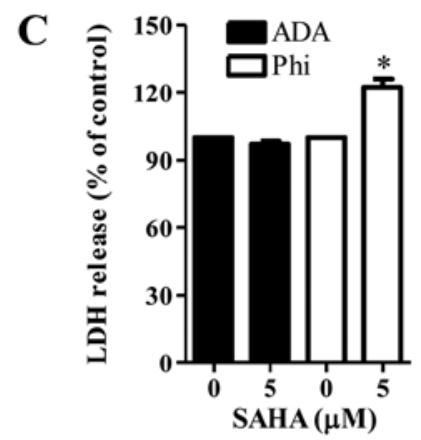

Phi

D ADA
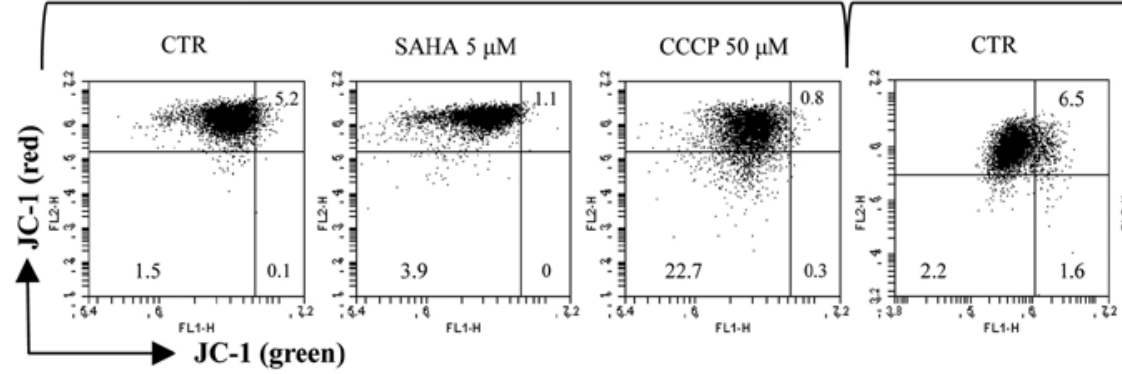

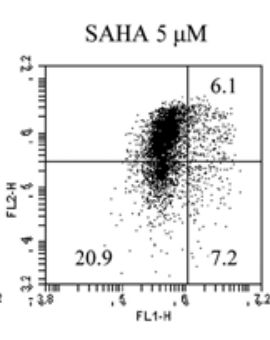

Figure 2. Effects of SAHA on cell death and mitochondrial membrane potential (MMP; $\triangle \Psi \mathrm{m}$ ) in ADA and Phi cells. (A) Graph shows the percent of Annexin V-FITC positive cells. (B) The protein levels of PARP, cleaved PARP, cleaved caspase-3 and $\beta$-actin in ADA and Phi cells. (C) Graph shows LDH release. (D) Each figure is a representative of JC-1 staining in SAHA-treated ADA and Phi cells. * $\mathrm{P}<0.05$ compared with SAHA-untreated control group.
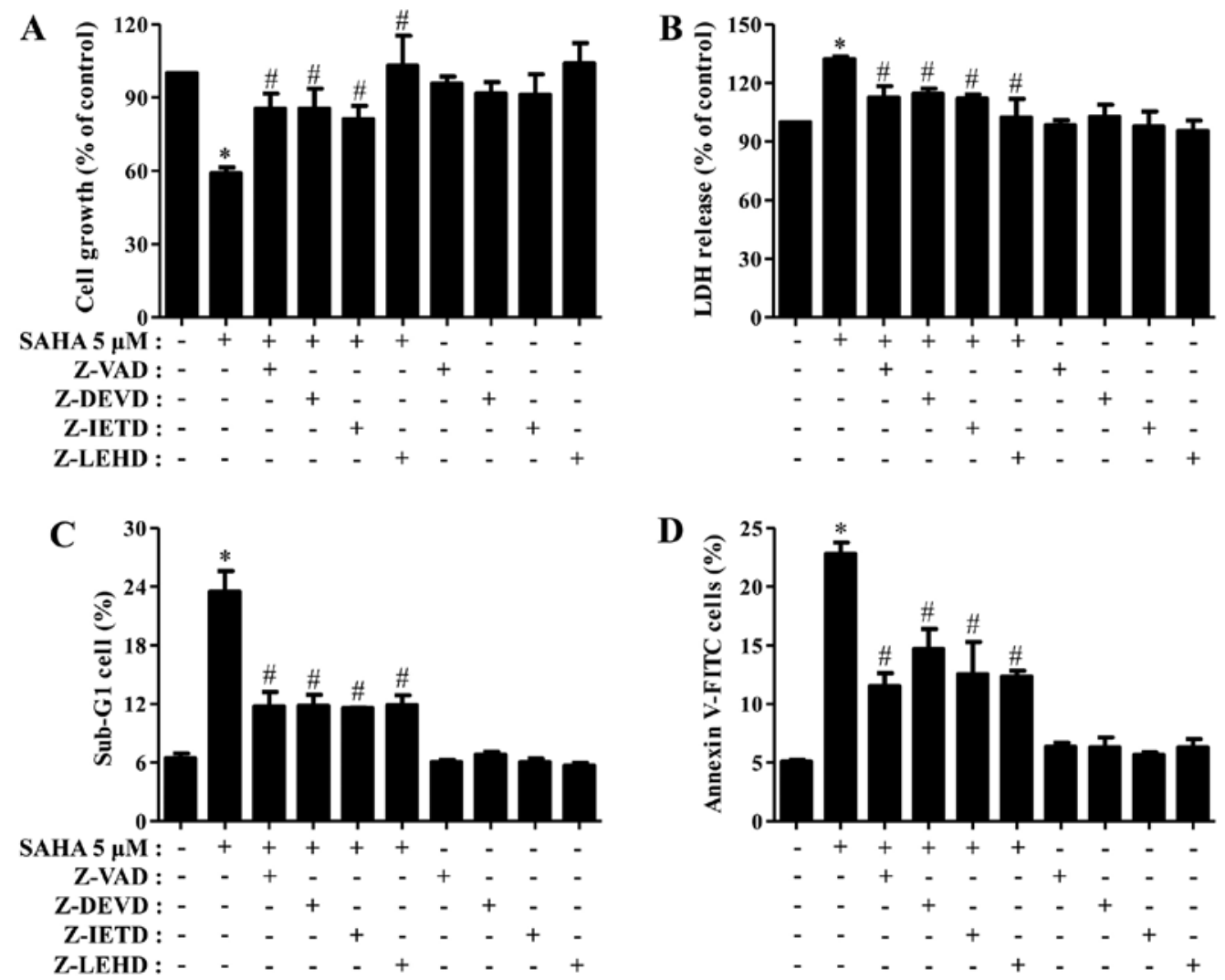

Figure 3. Effects of each caspase inhibitor on cell growth, LDH release and cell death in Phi cells. Graphs show (A) cell growth and (B) LDH release. Graphs show the (C) percent of sub-G1 and (D) Annexin V-FITC positive cells. ${ }^{*} \mathrm{P}<0.05$ compared with SAHA-untreated control group. ${ }^{\#} \mathrm{P}<0.05$ compared with cells treated with SAHA only.

(Fig. 2D). Carbonyl cyanide m-chlorophenyl hydrazine (CCCP) was used as a positive control to induce the loss of $\Delta \Psi_{\mathrm{m}}$.
Next, we determined which caspase is involved in Phi cell death and growth inhibition induced by SAHA. As shown 
A

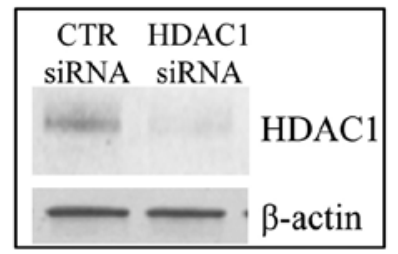

B

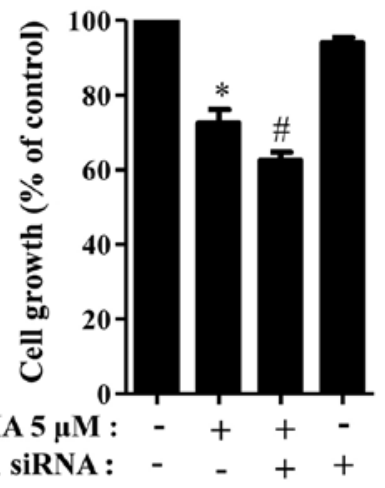

C

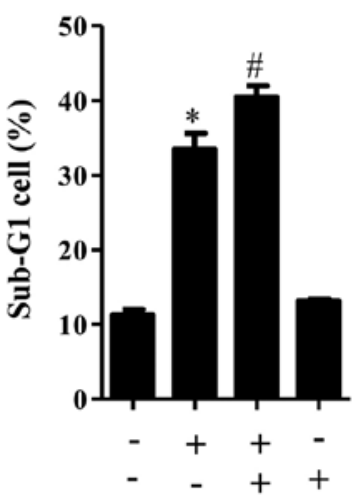

D

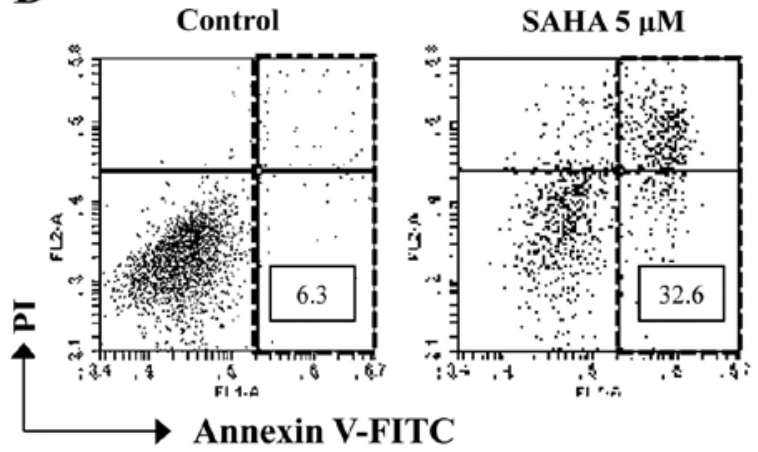

SAHA $5 \mu$ M + HDAC1 SIRNA

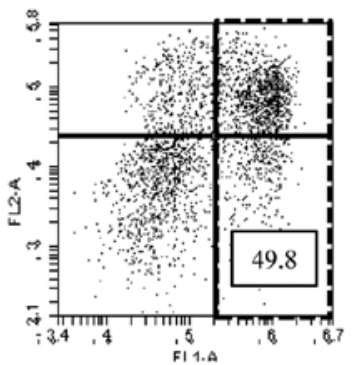

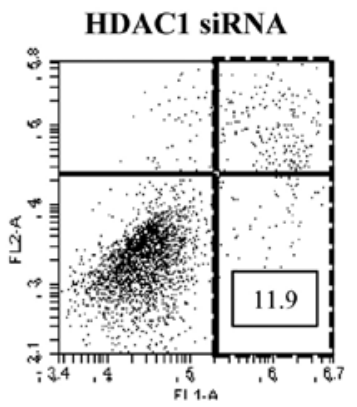

Figure 4. Effects of HDAC1 siRNA on cell growth and cell death in SAHA-treated Phi cells. (A) The expression level of HDAC1 was examined by western blot analysis. Graphs show (B) cell growth and the (C) percent of sub-G1 cells. (D) Each figure is a representative of Annexin V-FITC/PI staining cells. ${ }^{*} \mathrm{P}<0.05$ compared with SAHA-untreated control group. ${ }^{\#} \mathrm{P}<0.05$ compared with cells treated with SAHA only.

Fig. 3A and B, all the tested caspase inhibitors partially recovered the growth inhibition and LDH release of SAHA-treated Phi cells. In addition, all the inhibitors strongly reduced the percents of sub-G1 cells and Annexin V-FITC cells in these cells (Fig. 3C and D).

Effects of HDACl siRNA on cell growth and death in SAHAtreated Phi cells. Because the basal levels of HDAC1 were different between SAHA-sensitive and SAHA-resistant mesothelioma cells (Figs. 1 and 2), the status of HDAC1 might influence mesothelioma cell death caused by SAHA. To investigate whether HDAC1 protein affects SAHA-induced cell death in mesothelioma, the mRNA level of HDAC1 was knocked downed by the administration of siRNA. The knockdown of HDAC1 successfully occurred in Phi cells via its siRNA (Fig. 4A). HDAC1 siRNA significantly promoted cell growth inhibition in SAHA-treated Phi cells (Fig. 4B). In addition, HDAC1 siRNA increased the numbers of sub-G1 cells and Annexin V-positive cells in these cells (Fig. 4C and D). HDAC1 siRNA alone induced cell death in SAHA-untreated Phi control cells (Fig. 4D).

Effects of SAHA on intracellular ROS and Trxl levels in SAHA-treated Phi cells. When we measured the intracellular ROS levels by DCFDA and DHE dyes, $5 \mu \mathrm{M}$ SAHA significantly increased ROS levels including $\mathrm{O}_{2}{ }^{-}$in Phi cells (Fig. 5A). Moreover, well-known antioxidants, NAC and Vit.C effectively blocked cell growth inhibition and LDH release in SAHA-treated Phi cells (Fig. 5B and C) and both of them significantly reduced the number of Annexin V-positive cells in the cells (Fig. 5D).

It is reported that SAHA decreased Trx1 in cancer cells (24). Cellular antioxidants can change intracellular ROS levels and affect cell death such as apoptosis. As shown in Fig. 6A and $\mathrm{B}, 5 \mu \mathrm{M}$ SAHA decreased the mRNA and protein levels of Trx1 in Phi and ROB cells. While SAHA decreased the protein level of Trx1 in REN cells, this agent did not alter the mRNA level of Trx1 in these cells (Fig. 6A and B). Next, it was determined whether Trx1 knockdown affects cell growth, cell death and ROS levels in SAHA-treated Phi cells. Administration of Trx1 siRNA markedly decreased the level of Trx1 (Fig. 6C) and this siRNA enhanced cell growth inhibition in SAHA-untreated and -treated Phi cells (Fig. 6D). Trx1 siRNA also increased the percents of sub-G1 cells and apoptotic cells in these cells (Fig. 6E and F). Furthermore, Trx1 siRNA increased the $\mathrm{O}_{2}{ }^{-}$levels in SAHA-untreated and -treated Phi cells (Fig. 6G).

\section{Discussion}

Mesothelioma is a rare form of cancer derived from the pleura, peritoneum or pericardium. Epigenetic changes affect drug resistance and pathogenesis of mesothelioma. In the present study, we determined an anticancer effect of SAHA on various mesothelioma cells in view of HDAC1 and Trx1 levels. SAHA inhibited the growth of Phi and ROB mesothelioma cells. This result supports that HDAC inhibitor shows anti-tumor effect on mesothelioma $(9,25)$. However, this drug did not affect the 
A

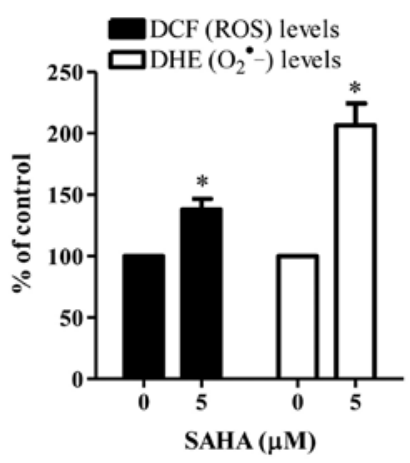

C

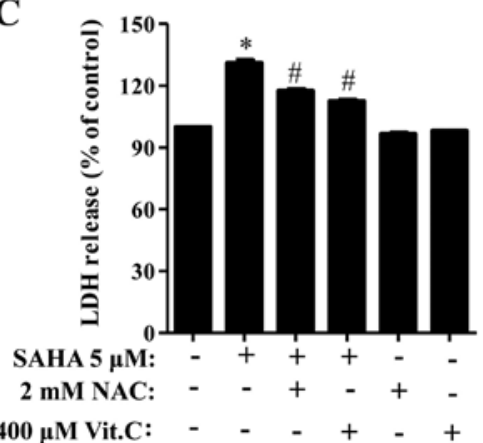

B

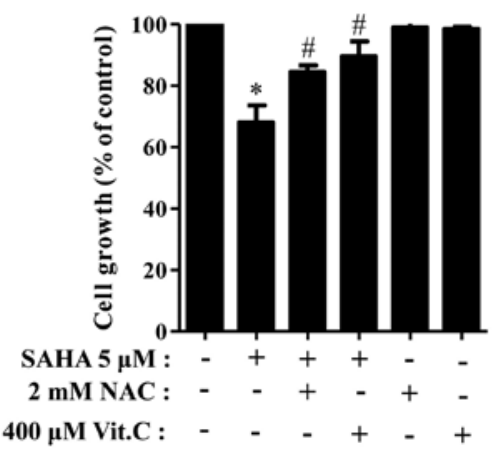

D

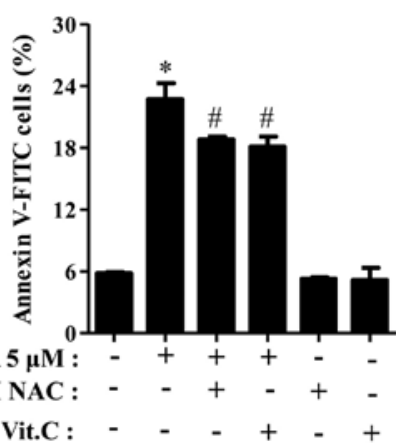

Figure 5. Effects of NAC and Vit.C on cell growth and cell death in SAHA-treated Phi cells. (A) Graph shows the intracellular ROS (black bar) and $\mathrm{O}_{2}{ }^{-}$(white bar) levels. Graphs show (B) cell growth, (C) LDH release and (D) the percent of Annexin V-FITC positive cells in Phi cells. "P<0.05 compared with SAHAuntreated control group. ${ }^{.} \mathrm{P}<0.05$ compared with cells treated with SAHA only.

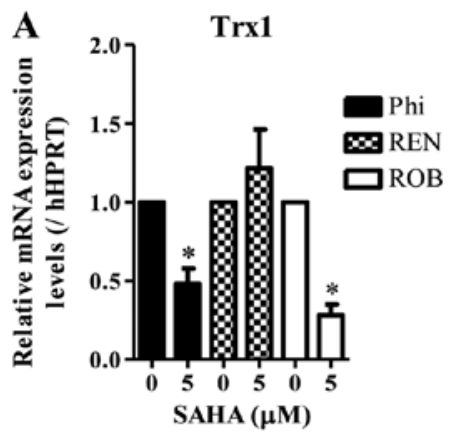

B

D

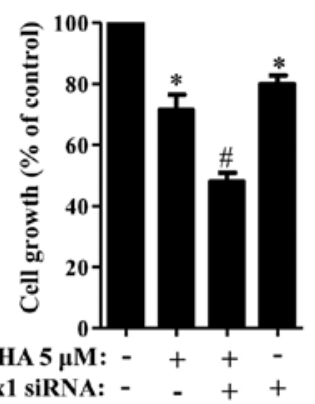

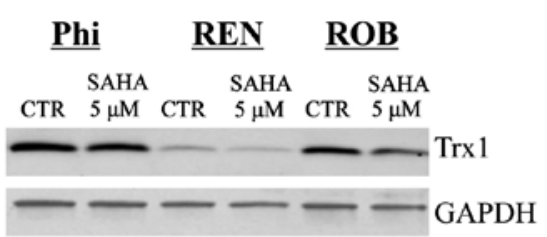

C

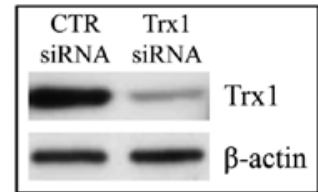

G

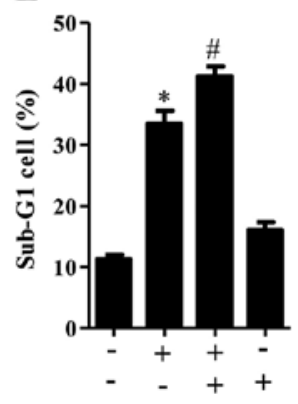

F

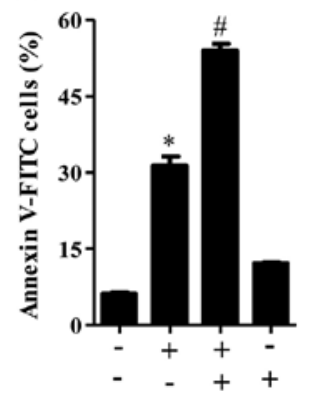

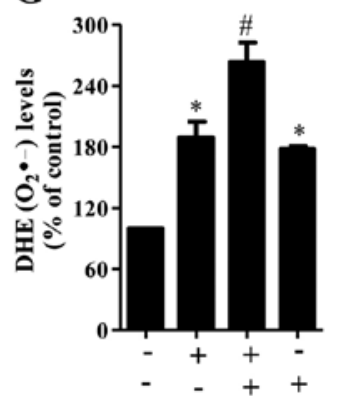

Figure 6. Effects of Trx1 siRNAs on cell growth, cell death and $\mathrm{O}_{2}^{-}$level and in SAHA-treated Phi cells. (A) The mRNA and (B) protein levels of Trx1 in SAHA-treated Phi, REN and ROB cells. (C) The expression level of Trx1 was examined by western blot analysis. Graphs show (D) cell growth and the (E) percent of sub-G1 cells. Graphs show (F) the percent of Annexin V-FITC positive cells and intracellular $\mathrm{O}_{2}{ }^{-}$level $(\mathrm{G})$ in Phi cells. " $\mathrm{P}<0.05$ compared with SAHA-untreated control group. ${ }^{\text {} P}<0.05$ compared with cells treated with SAHA only.

growth of ADA and Mill mesothelioma cells. Interestingly, SAHA-sensitive Phi and ROB cells strongly expressed the basal level of HDAC1 protein whereas SAHA-resistant ADA and Mill cells showed lower basal levels of HDAC1. Moreover, 
SAHA notably inhibited HDAC activity and induced acetylation of H4 in SAHA-sensitive Phi cells. This result implies that SAHA as an HDAC inhibitor especially works well in mesothelioma cells having overexpressed HDAC1 protein. In addition, the different levels of HDAC1 among mesothelioma cells seemed to differently influence the sensitivity of mesothelioma cells to HDAC inhibitor. Many studies have reported that inhibition of HDAC1 enhances cell death in various cancer cells including ovarian and liver $(26,27)$. Likewise, HDAC1 knockdown enhanced Phi cell death caused by SAHA. Taken together, HDAC1 might be a target for the mesothelioma therapy.

SAHA induced apoptosis in Phi cells which was accompanied by the cleavages of PARP and caspase- 3 and the loss of MMP $(\Delta \Psi \mathrm{m})$. In addition, all the tested caspase inhibitors strongly prevented cell death in these cells. Therefore, it seems that apoptosis occurs via intrinsic and extrinsic pathways and that cell death is the main mechanism for the inhibition of cell growth by SAHA. We also observed that SAHA induced LDH release in SAHA-treated Phi cells. However, NecroX-2 and necrostatin1, necrosis inhibitors did not significantly attenuate cell death in SAHA-treated Phi cells (data not shown). Therefore, necrosis seems to be in part related to SAHAinduced Phi cell death.

Oxidative stress is an important cause in mesothelioma cell death and antioxidant contributes the drug resistance of mesothelioma cells. Likewise, SAHA increased the intracellular ROS levels including $\mathrm{O}_{2}{ }^{--}$in Phi cells. Both NAC and Vit.C prevented the growth inhibition and cell death in SAHAtreated Phi cells. SAHA did not change ROS level in ADA cells which was resistant to this drug (data not shown). Therefore, these results suggest that oxidative stress induced by SAHA leads to apoptotic cell death in Phi mesothelioma cells.

Trx is an important antioxidant protein in cells and it protects the cell from oxidative stress damage by facilitating the reduction of other oxidative proteins via cysteine thioldisulfide exchange (28). HDAC inhibitor changes the redox state of Trx (29). In the present study, SAHA downregulated the mRNA and protein levels of Trx1 in Phi and ROB cells. In addition, Trx1 siRNA sensitized Phi cells to SAHA and it alone induced apoptosis in SAHA-untreated Phi cells. These results imply that Trx1 has a critical role in cell death in mesothelioma cells. In regard to ROS levels, Trx1 siRNA intensified the $\mathrm{O}_{2}{ }^{-}$level in SAHA-treated and untreated Phi cells. This result suggests that Trx 1 also act as a strong antioxidant in mesothelioma cells.

In conclusion, SAHA inhibited the growth of Phi and ROB cells among the tested human mesothelioma cells. These cells relatively have higher levels of HDACs. SAHA-induced Phi cell death was related to oxidative stress and Trx1 levels.

\section{Acknowledgements}

We thank Professor Peter R. Hoffmann and Dr Pietro Bertino for providing mesothelioma cells. The present study was supported by the National Research Foundation of Korea (NRF) grant funded by the Korea government (MSIP) (no. 2008-0062279) and supported by the Basic Science Research Program through the NRF funded by the Ministry of Education (2013006279).

\section{References}

1. Comertpay S, Pastorino S, Tanji M, Mezzapelle R, Strianese O, Napolitano A, Baumann F, Weigel T, Friedberg J, Sugarbaker P, et al: Evaluation of clonal origin of malignant mesothelioma. $\mathrm{J}$ Transl Med 12: 301, 2014.

2. Carbone M and Yang H: Molecular pathways: Targeting mechanisms of asbestos and erionite carcinogenesis in mesothelioma. Clin Cancer Res 18: 598-604, 2012.

3. Matsuzaki H, Maeda M, Lee S, Nishimura Y, Kumagai-Takei N, Hayashi H, Yamamoto S, Hatayama T, Kojima Y, Tabata R, et al: Asbestos-induced cellular and molecular alteration of immunocompetent cells and their relationship with chronic inflammation and carcinogenesis. J Biomed Biotechnol 2012: 492608, 2012.

4. Christensen BC, Houseman EA, Godleski JJ, Marsit CJ, Longacker JL, Roelofs CR, Karagas MR, Wrensch MR, Yeh RF, Nelson HH, et al: Epigenetic profiles distinguish pleural mesothelioma from normal pleura and predict lung asbestos burden and clinical outcome. Cancer Res 69: 227-234, 2009.

5. Karczmarski J, Rubel T, Paziewska A, Mikula M, Bujko M, Kober P, Dadlez M and Ostrowski J: Histone H3 lysine 27 acetylation is altered in colon cancer. Clin Proteomics 11: 24, 2014.

6. Kalari S, Moolky N, Pendyala S, Berdyshev EV, Rolle C, Kanteti R, Kanteti A, Ma W, He D, Husain AN, et al: Sphingosine kinase 1 is required for mesothelioma cell proliferation: Role of histone acetylation. PLoS One 7: e45330, 2012.

7. Kim NH, Kim SN and Kim YK: Involvement of HDAC1 in E-cadherin expression in prostate cancer cells; its implication for cell motility and invasion. Biochem Biophys Res Commun 404: 915-921, 2011.

8. Hayashi A, Horiuchi A, Kikuchi N, Hayashi T, Fuseya C, Suzuki A, Konishi I and Shiozawa T: Type-specific roles of histone deacetylase (HDAC) overexpression in ovarian carcinoma: HDAC1 enhances cell proliferation and HDAC3 stimulates cell migration with downregulation of E-cadherin. Int J Cancer 127: 1332-1346, 2010.

9. Paik PK and Krug LM: Histone deacetylase inhibitors in malignant pleural mesothelioma: Preclinical rationale and clinical trials. J Thorac Oncol 5: 275-279, 2010.

10. Katafygiotis P, Giaginis C, Patsouris E and Theocharis S: Histone deacetylase inhibitors as potential therapeutic agents for the treatment of malignant mesothelioma. Anticancer Agents Med Chem 13: 476-482, 2013.

11. Schieber M and Chandel NS: ROS function in redox signaling and oxidative stress. Curr Biol 24: R453-R462, 2014.

12. Tabata C, Terada T, Tabata R, Yamada S, Eguchi R, Fujimori Y and Nakano T: Serum thioredoxin-1 as a diagnostic marker for malignant peritoneal mesothelioma. J Clin Gastroenterol 47: e7-e11, 2013.

13. Thompson JK, Westbom CM, MacPherson MB, Mossman BT, Heintz NH, Spiess P and Shukla A: Asbestos modulates thioredoxin-thioredoxin interacting protein interaction to regulate inflammasome activation. Part Fibre Toxicol 11: 24, 2014

14. Murthy S, Adamcakova-Dodd A, Perry SS, Tephly LA, Keller RM, Metwali N, Meyerholz DK, Wang Y, Glogauer M, Thorne PS, et al: Modulation of reactive oxygen species by Rac1 or catalase prevents asbestos-induced pulmonary fibrosis. Am J Physiol Lung Cell Mol Physiol 297: L846-L855, 2009.

15. Powis G and Kirkpatrick DL: Thioredoxin signaling as a target for cancer therapy. Curr Opin Pharmacol 7: 392-397, 2007.

16. Wang J, Yang H, Li W, Xu H, Yang X and Gan L: Thioredoxin 1 upregulates FOXO1 transcriptional activity in drug resistance in ovarian cancer cells. Biochim Biophys Acta 1852: 395-405, 2015.

17. Min A, Im SA, Kim DK, Song SH, Kim HJ, Lee KH, Kim TY, Han SW, Oh DY, Kim TY, et al: Histone deacetylase inhibitor, suberoylanilide hydroxamic acid (SAHA), enhances anti-tumor effects of the poly (ADP-ribose) polymerase (PARP) inhibitor olaparib in triple-negative breast cancer cells. Breast Cancer Res 17: 33, 2015.

18. Ding L, Zhang Z, Liang G, Yao Z, Wu H, Wang B, Zhang J, Tariq M, Ying $M$ and Yang B: SAHA triggered MET activation contributes to SAHA tolerance in solid cancer cells. Cancer Lett 356: 828-836, 2015.

19. Hurwitz JL, Stasik I, Kerr EM, Holohan C, Redmond KM, McLaughlin KM, Busacca S, Barbone D, Broaddus VC, Gray SG, et al: Vorinostat/SAHA-induced apoptosis in malignant mesothelioma is FLIP/caspase 8-dependent and HR23B-independent. Eur J Cancer 48: 1096-1107, 2012. 
20. Han YH, Kim SZ, Kim SH and Park WH: Pyrogallol inhibits the growth of lung cancer Calu- 6 cells via caspase-dependent apoptosis. Chem Biol Interact 177: 107-114, 2009.

21. You BR and Park WH: Gallic acid-induced lung cancer cell death is related to glutathione depletion as well as reactive oxygen species increase. Toxicol In Vitro 24: 1356-1362, 2010.

22. You BR, Kim SH and Park WH: Reactive oxygen species, glutathione, and thioredoxin influence suberoyl bishydroxamic acid-induced apoptosis in A549 lung cancer cells. Tumour Biol 36: 3429-3439, 2015.

23. You BR, Shin HR, Han BR and Park WH: PX-12 induces apoptosis in Calu-6 cells in an oxidative stress-dependent manner. Tumour Biol 36: 2087-2095, 2015.

24. Butler LM, Zhou X, Xu WS, Scher HI, Rifkind RA, Marks PA and Richon VM: The histone deacetylase inhibitor SAHA arrests cancer cell growth, up-regulates thioredoxin-binding protein-2, and down-regulates thioredoxin. Proc Natl Acad Sci USA 99: $11700-11705,2002$.

25. Crisanti MC, Wallace AF, Kapoor V, Vandermeers F, Dowling ML, Pereira LP, Coleman K, Campling BG, Fridlender ZG, Kao GD, et al: The HDAC inhibitor panobinostat (LBH589) inhibits mesothelioma and lung cancer cells in vitro and in vivo with particular efficacy for small cell lung cancer. Mol Cancer Ther 8: 2221-2231, 2009.
26. Xie HJ, Noh JH, Kim JK, Jung KH, Eun JW, Bae HJ, Kim MG, Chang YG, Lee JY, Park H, et al: HDAC1 inactivation induces mitotic defect and caspase-independent autophagic cell death in liver cancer. PLoS One 7: e34265, 2012.

27. Cacan E, Ali MW, Boyd NH, Hooks SB and Greer SF: Inhibition of HDAC1 and DNMT1 modulate RGS10 expression and decrease ovarian cancer chemoresistance. PLoS One 9: e87455, 2014.

28. Chae JS, Gil Hwang S, Lim DS and Choi EJ: Thioredoxin-1 functions as a molecular switch regulating the oxidative stressinduced activation of MST1. Free Radic Biol Med 53: 2335-2343, 2012.

29. Ungerstedt J, Du Y, Zhang H, Nair D and Holmgren A: In vivo redox state of human thioredoxin and redox shift by the histone deacetylase inhibitor suberoylanilide hydroxamic acid (SAHA). Free Radic Biol Med 53: 2002-2007, 2012. 